\title{
Competition with Open Spectrum Access: Sharing vs. Unlicensed Access
}

\author{
Chang Liu \\ EECS Department \\ Northwestern University, Evanston, IL 60208 \\ Email: changliu2012@u.northwestern.edu
}

\author{
Randall A. Berry \\ EECS Department \\ Northwestern University, Evanston, IL 60208 \\ Email: rberry@eecs.northwestern.edu
}

\begin{abstract}
We consider two scenarios for adding open access spectrum to increase competition in a wireless service market: making new spectrum available for unlicensed use or requiring an incumbent license holder to share it with secondary providers. Our main result is that social welfare and consumer welfare with shared spectrum are always greater than with unlicensed access. Also when the amount of spectrum is large enough, the primary service provider gains more profit with sharing than with unlicensed access.
\end{abstract}

\section{INTRODUCTION}

Due in part to the success of WiFi, there has been much interest in expanding the amount of spectrum available for open access. In particular, making "prime" spectrum available for such use has the potential of lowering the barriers required for new entrants to offer wireless services. Here, we consider two approaches for allowing for such open access: sharing spectrum or allocating a separate unlicensed band. For each we study the effect of competition among new entrants and incumbents on economic welfare.

With spectrum sharing, we consider the well-known "primary-secondary" approach [4] in which secondary users can access spectrum provided they do not interfere with primary users. This can be facilitated by sensing [1], a database approach [2] or a market-based approach [3]. Here, we consider a scenario in which the primary user is an incumbent service provider and thus competes with the secondary entrants for customers. Alternatively, we consider a scenario in which new spectrum is set aside for unlicensed access. In this case, all new entrants can use the unlicensed band, and no user of this band is prioritized over any other. The incumbent provider retains an exclusive license to its own band.

In both cases, lowering entry barriers will increase competition and so can potentially increase social welfare. However, the low cost of entry may also lead to the spectrum becoming overcrowded, i.e., a "tragedy of the commons." Indeed, in [6], a model for adding unlicensed spectrum to a market was studied showing that due to congestion, a type of Baress's paradox [12] can occur in which social welfare can decrease with the addition of new spectrum. Here, we use a similar model for unlicensed access (see also [?], [9], [10]) and a related model developed in [5] for shared spectrum. These models in turn

This research is supported in part by NSF grants CNS-1147786, SES1247984 and SES-1343381. are related to work in the operations management literature on competition with congestible resources (e.g. [7], [8]).

We compare the resulting social welfare, consumer welfare and firm profits with sharing (as in [5]) and unlicensed access (as in [6]). To normalize the total amount of spectrum available in both cases, we assume that the same amount of additional spectrum is added to the market, where in the sharing case this is given to the primary, who then must share all of the available spectrum. In both models, the firms compete for the same pool of customers by offering a service price. Customers then choose firms based on a delivered price, which consists of the announced service price plus a congestion cost, representing the interference from each user in the network. With shared spectrum, primary users will not see any interference from secondary users while secondary users "see" not only themselves but also primary users. In contrast, in unlicensed bands, no such priority exists.

Our analysis shows that sharing results in greater social welfare and consumer welfare than unlicensed access and potentially more firm profit as well, provided the extra bandwidth is large enough. As extra bandwidth increases, the social and consumer welfare from sharing also increases while firm profit decreases. In contrast, unlicensed access results in decreasing welfares within a certain range but then they increase.

The rest of the paper is organized as follows. Models for price competition with sharing and unlicensed access are described in Section II. We present an analysis of the case of linear congestion costs and homogeneous inverse-demand in Section III. We then deal with more general congestion costs and demand in Section IV. Finally, we conclude in Section V.

\section{Competition Model}

We consider a wireless service market where service providers (SPs) set prices for their services and compete for a common pool of customers as in [6]. The consumers respond according to the delivered price, given by the sum of the service price and congestion cost of a SP. We focus on a model in which there is one incumbent SP with licensed spectrum bandwidth $C$. Initially this firm acts as a monopolist. Additional spectrum with bandwidth $B$ is to be added to the market either as shared spectrum or unlicensed spectrum, and it will be used by $N>1$ new entrants, $S_{1}, S_{2}, \ldots, S_{N}$, who all compete with the incumbent for a common pool of customers. 
With sharing, the incumbent SP will be the primary spectrum user for the entire bandwidth of $B+C$, while the entrants can use this subject to not degrading the performance of the primary. With unlicensed access, the entrants can only access the common unlicensed band.

The service that SPs offer is characterized by a congestion cost, $g(x)$, which models various congestion effects such as increased interference or queueing delays that arise as a SP serves more customers in a given area. With sharing, the congestion cost seen by the primary SP's customers is given by $g\left(x_{1} /(B+C)\right)$, where $x_{1}$ denotes the mass of customers the primary is serving. Customers of the secondary SPs will encounter a congestion cost of $g\left(X^{S} /(B+C)\right)$, where

$$
X^{S}=x_{1}+\sum_{i=1}^{N} x_{i}^{S} .
$$

Here, $X^{S}$ is the overall mass of customers being served in the licensed band with $x_{n}^{S}$ being the number of customers served by the $n t h$ new entrant. This models the fact that the primary SP does not "see" any degradation from the secondaries, while the secondary users experience degradation due to the primary as well as each other. In case of unlicensed spectrum, the congestion cost seen by the new entrants is $g\left(X^{U} / B\right)$, where

$$
X^{U}=\sum_{i=1}^{N} x_{i}^{S}
$$

Here $X^{U}$ is the overall mass of customers being served in the unlicensed band. This models the fact that with unlicensed spectrum entrants only experience interference from themselves. In general we assume that $g(x)$ is an increasing, convex function as shown in Fig. 1, though for much of our analysis, for simplicity, we focus on the linear case, i.e., $g(x)=x$.

Customers select SPs based on the delivered price for each SP's service, which is the sum of the SPs' announced price and the congestion cost. Specifically, the delivered price of the primary SP with announced price $p_{1}$ is $p_{1}+g\left(x_{1} /(B+\right.$ $C)$ ) for sharing and $p_{1}+g\left(x_{1} / C\right)$ for unlicensed access. The delivered price of each new entrant $S_{i}$, with announced price $p_{i}^{S}$ is $p_{i}^{S}+g\left(X^{S} /(B+C)\right)$ for sharing and $p_{i}^{S}+g\left(X^{U} / B\right)$ for unlicensed access. Each customer selects the service provider who has the lowest delivered price. If more than one SP has the same delivered price, a customer chooses one of the low price SPs uniformly at random.

We assume a single mass of infinitesimal customers, and we normalize the total customer mass to be 1 . Customer demand is modeled by an inverse demand function, $P(q)$, which is a non-increasing function, that indicates the delivered price at which a mass of $q$ customers are willing to pay for service with price $P(q)$. As shown in Fig. 1, this will in general be a concave function. In Section III, we assume that customers are homogeneous in their demand, i.e., all customers are willing to pay up to the same delivered price, in which case $P(q)$ has a "box" shape. Then in Section IV, we consider the general case and give an example of heterogeneous demand, where $P(q)=1-q$, for $q$ between 0 and 1 , and $P(q)=0$, otherwise.
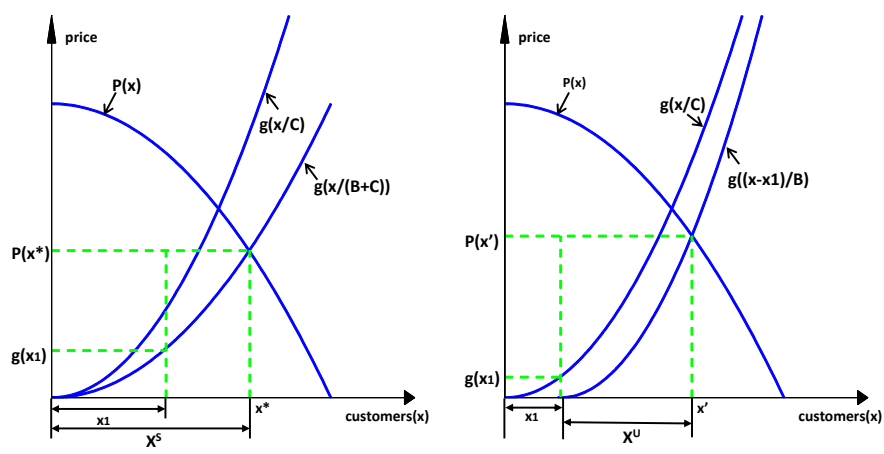

Fig. 1. Illustration of the pricing game. Left: with shared spectrum. Right: with unlicensed spectrum.

We view the competition among the incumbent SP and the new entrant SPs as a pricing game where firms set prices, $\left(p_{1}, \mathbf{p}^{\mathbf{S}}\right)$, simultaneously, where $\mathbf{p}^{\mathbf{S}}=\left(p_{1}^{S}, \ldots, p_{N}^{S}\right)$. Customers then choose one of the firms offering the lowest delivered price. Thus, given a set of prices, $\left(p_{1}, \mathbf{p}^{\mathbf{S}}\right)$, the customers receiving service must satisfy Wardrop equilibrium conditions [11], which specify that the delivered price of all SPs serving customers are equal and no greater than the inverse demand evaluated at the total number of customers served. For example, in the case of sharing theses conditions become:

$$
\begin{array}{rlr}
p+g\left(x_{1} /(B+C)\right) & \leq P\left(X^{S}\right), & \text { (with equality if } \left.x_{1}>0\right) \\
p_{i}^{S}+g\left(X^{S} /(B+C)\right) & =P\left(X^{S}\right), & \text { for } i \in N \text { with } x_{i}^{S}>0, \\
p_{i}^{S}+g\left(X^{S} /(B+C)\right) & \geq P\left(X^{S}\right), & \forall i \in N .
\end{array}
$$

We define a (pure strategy) Nash equilibrium of the overall pricing game to be a set of prices $\left(p_{1}, \mathbf{p}^{\mathbf{S}}\right)$ and demands $\left(x_{1}, \mathbf{x}^{\mathbf{S}}\right)$, which satisfy these Wardrop equilibrium conditions and also have the property that no SP can increase its profit by unilaterally changing its price.

Given such an equilibrium, the firm profit, $f_{c}$ is the sum of the profits made by all SPs. The welfare of the $x$ th consumer served is the difference between that consumer's value for the service (given by $P(x)$ ) and the delivered price of the service; consumers that are not served receive zero welfare. The total consumer welfare, $S_{c}$, is the integral of this over all consumers. The social welfare, $S$, of the entire economy is the sum of the firm profit and the consumer welfare, i.e.,

$$
S=S_{c}+f_{c}
$$

Next we give some preliminary discussions of this game both with shared and unlicensed spectrum.

\section{A. Shared Spectrum}

Similar to [5], since all secondary SPs are offering the same service, it can be shown in any equilibrium they must all be offering a price $p_{i}^{S}=0$. In that case, the delivered price for each secondary SP is simply the congestion cost, which must be equal to the inverse demand, i.e.,

$$
g\left(x^{*} /(B+C)\right)=P\left(x^{*}\right) .
$$


Here $x^{*}$ is the total customer mass in the shared band (see the left-hand side of Fig. 1). The primary firm then maximizes its profit by solving:

$$
\begin{array}{ll}
\max & p x_{1} \\
\text { subject to } & p+g\left(x_{1} /(B+C)\right)=P\left(x^{*}\right), \\
& g\left(x^{*} /(B+C)\right)=P\left(x^{*}\right), \\
& 0 \leq x_{1} \leq 1 .
\end{array}
$$

As $x^{*}$ is determined only by (1), $P\left(x^{*}\right)$ is fixed, given the congestion and demand functions.

This optimization problem is a convex problem, which has a unique solution. Before the additional spectrum is added, the incumbent's profit maximization is given by a similar problem with $B=0$ and without the constraint in (1).

\section{B. Unlicensed Spectrum}

As shown in [6], with unlicensed spectrum, all the entrant SPs will again offer $p=0$ in equilibrium. It follows that the delivered price equals the congestion cost in the unlicensed band, i.e.,

$$
g\left(\left(x^{\prime}-x_{1}\right) / B\right)=P\left(x^{\prime}\right),
$$

where $x_{1}$ is the number of users served by the incumbent and $x^{\prime}$ is the total number of users served by any SP in the market (see the right-hand side of Fig. 1). The primary firm then maximizes its revenue by solving:

$$
\begin{array}{ll}
\max & p x_{1} \\
\text { subject to } & p+g\left(x_{1} / C\right)=P\left(x^{\prime}\right), \\
& g\left(\left(x^{\prime}-x_{1}\right) / B\right)=P\left(x^{\prime}\right), \\
& 0 \leq x_{1} \leq 1 .
\end{array}
$$

Again this is a convex problem with a unique solution. Note that compared to the sharing case, here the primary can influence the delivered price, in particular by changing its price and the number of customers it serves, it will shift the location of the $g\left(\left(x^{\prime}-x_{1}\right) / B\right)$ curve in Fig. 1 .

\section{Welfare ANALYSis With LINEAR CONGESTION AND HOMOGENEOUS INVERSE DEMAND}

In this section, we compare shared and unlicensed spectrum when the congestion cost is linear (i.e, $g(x)=x$ ) and the customer demand is homogeneous (i.e., $P(x)=1$ when $0 \leq$ $x \leq 1$ and $P(x)=0$, elsewhere). Given such conditions, we characterize the social welfare, $S$, consumer welfare, $S_{c}$, and firm profit, $f_{c}$ both with shared and unlicensed spectrum. We first summarize our main result in the following theorem:

Theorem 3.1: Social welfare and consumer welfare with sharing increases with additional spectrum bandwidth $B$ and are always no less than that with unlicensed access. When $B$ is large enough, the primary firm can gain more profit with sharing than with unlicensed access.

We will prove this theorem by first specifying the welfares in both regulation scenarios and then comparing their behaviors as a function of $B$.

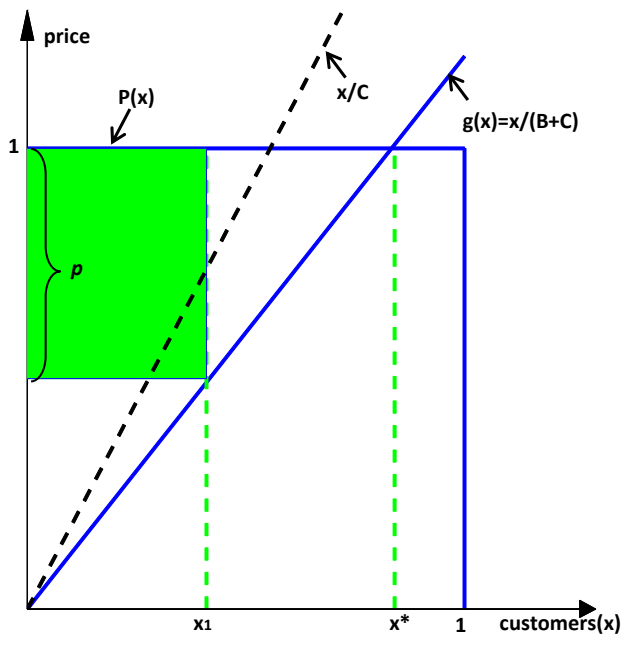

Fig. 2. Illustration of spectrum sharing competition game with linear congestion and homogeneous inverse-demand when $B+C \leq 1$.

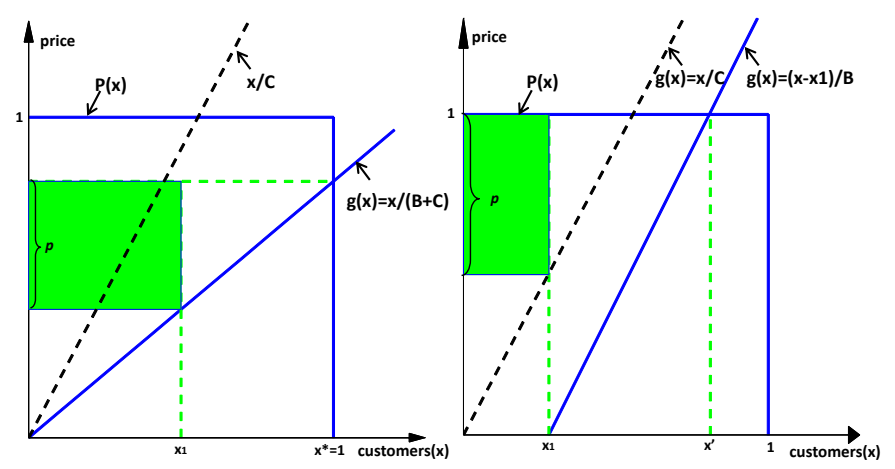

Fig. 3. Illustration of competition game with linear congestion and homogeneous inverse-demand. Left: shared spectrum when $B+C>1$. Right: unlicensed spectrum when $0<B \leq 1-C / 2$.

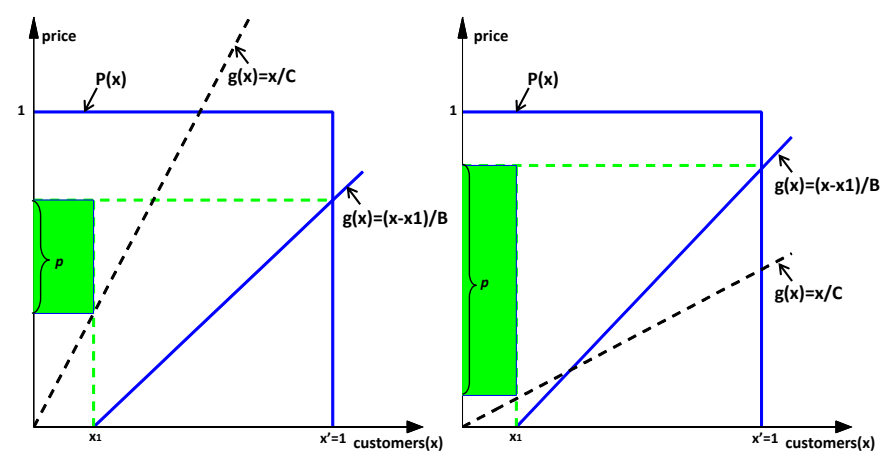

Fig. 4. Illustration of competition game with linear congestion and homogeneous inverse-demand. Left: unlicensed spectrum when $1-C / 2<$ $B \leq\left(\sqrt{1+C^{2}}-C+1\right) / 2$. Right: unlicensed spectrum when $B>$ $\left(\sqrt{1+C^{2}}-C+1\right) / 2$.

\section{A. Welfare With Sharing}

Due to the discontinuity of $P(x)$, we separate our discussion into two parts. When $B$ and $C$ are both relatively small, due to high congestion it is not possible to serve all the customers, even with a price of zero. In this situation, the delivered price 
from the primary SP is only limited by the maximum the customers are willing to pay, i.e., $P(x)=1$ (see Fig. 2). However, when $B$ grows larger, spectrum is no longer as scarce. In this case, competition will force the primary SP to lower its delivered price. Hence, all SPs will offer lower prices resulting in all customers being served but a lower profit for the primary SP (see the left-hand side of Fig. 3). This is summarized in the following lemma.

Lemma 3.2: With shared spectrum, linear congestion and homogeneous inverse-demand,

i) When $0<B+C \leq 1, S=f_{c}=(B+C) / 4, S_{c}=0$.

ii) When $B+C>1$,

$$
\begin{array}{r}
S=1-\frac{3}{4(B+C)}, \\
f_{c}=\frac{1}{4(B+C)}, \\
S_{c}=1-\frac{1}{(B+C)} .
\end{array}
$$

This shows that with sharing, social welfare increases with additional bandwidth $B$. Consumers will not see any welfare gains when the total spectrum available is limited but their welfare will continuously increase when $B+C$ grows large. Moreover, firm profit for the primary firm grows first but then decreases asymptotically to zero as the bandwidth increases. The decrease in firm profit comes from the competition from secondary firms as their existence decreases the delivered price.

\section{B. Welfare With Unlicensed Spectrum}

The effect on total welfare of adding unlicensed spectrum to a market was studied in [6]. We summarize these results for the model considered here. Similar to shared spectrum, when $B$ is small, not all demands are met as in Fig. 3. Note that in the unlicensed band, users are not subject to avoiding interference with the incumbent, but do mutually interfere with each other. When the additional bandwidth is large enough as in Fig. 4, some customers in the licensed band may shift to the unlicensed band thus creating more congestion there. A detailed summary of the welfare in this case is given in the following lemma.

Lemma 3.3: With unlicensed spectrum, linear congestion and homogeneous inverse-demand,

i) When $0<B \leq \max \left\{1-\frac{C}{2}, 0\right\}, S=f_{c}=C / 4, S_{c}=0$.

ii) When $\max \left\{1-\frac{C}{2}, 0\right\}<B \leq \frac{\sqrt{1+C^{2}}-C+1}{2}, S=f_{c}=$ $(1-B)\left(1-\frac{1-B}{C}\right)$ and $S_{c}=0$.

iii) When $B>\frac{\sqrt{1+C^{2}}-C+1}{2}$,

$$
\begin{aligned}
& S=1-\frac{4 B+C}{4 B(B+C)}, \\
& f_{c}=\frac{C}{4 B(B+C)}, \\
& S_{c}=1-\frac{2 B+C}{2 B(B+C)} .
\end{aligned}
$$
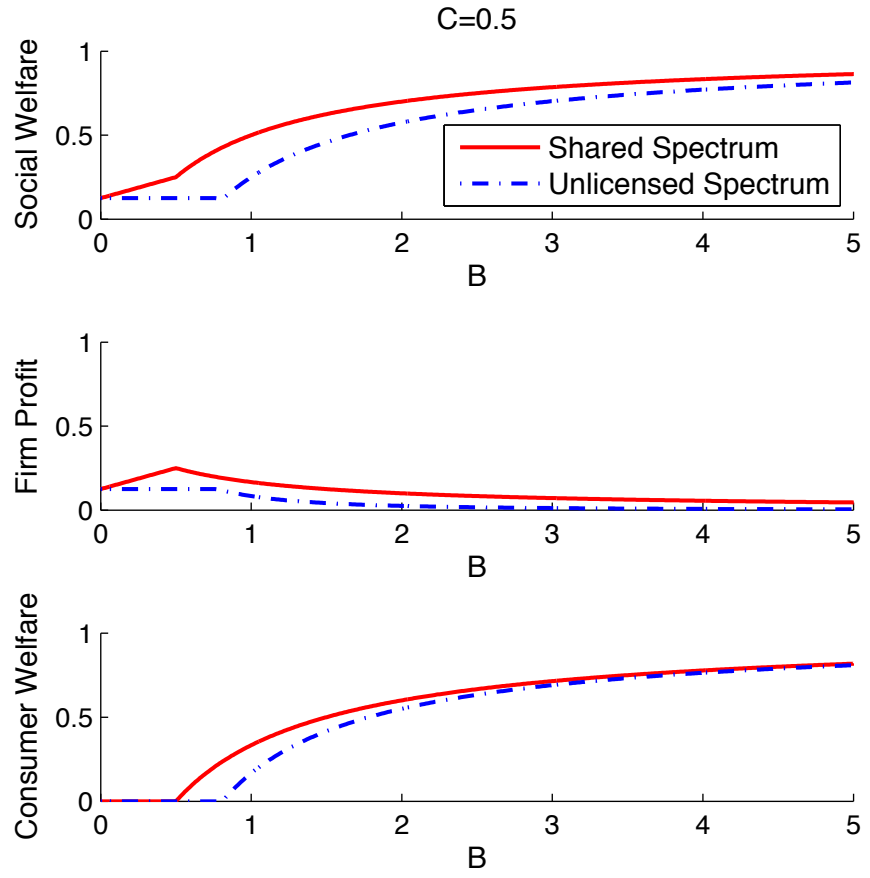

Fig. 5. Welfare comparison between sharing and unlicensed access with additional bandwidth $B$ when $C=0.5$.

In Fig. 5-7, the welfare for both licensed and shared spectrum is plotted as a function of $B$ for different values of $C$. As shown, the initial "gap" with unlicensed access in total welfare increases with $C$. Moreover, when the incumbent has limited bandwidth, i.e., $C$ is small as in Fig. 5, total welfare, consumer welfare and firm profit with sharing are greater than with unlicensed access for all $B$ and they both asymptotically approach each other when $B$ becomes large. In fact, this is true for social welfare and consumer welfare no matter how large $C$ is. However, when the incumbent has more bandwidth initially, firms are better off with unlicensed access within a certain range. And as $B$ grows large enough, the difference becomes negative. The initial welfares of the two cases in the plots are different because with sharing, when $B=0$, we are plotting the result still assuming that the original spectrum is shared, while with unlicensed spectrum, there is no spectrum for the entrants when $C=0$. In Fig. 5 and 6 , social welfare from unlicensed spectrum does not change for small $B$; however, Fig. 7 shows that with large initial bandwidth $C$, social welfare will decrease immediately after adding additional unlicensed bandwidth until the worst case when $B=\frac{\sqrt{1+C^{2}}-C+1}{2}$. The range of $B$ for which welfare is decreasing, decreases with $C$ so that the largest range occurs when $C=2$.

\section{Welfare Analysis with General Congestion AND GENERAL DEMAND}

Previously we considered the case of linear congestion costs and homogeneous inverse-demand with shared and unlicensed spectrum. Now we consider a general setting with $g(x)$ being strictly increasing while $P(x)$ is strictly decreasing in $x$. Our 

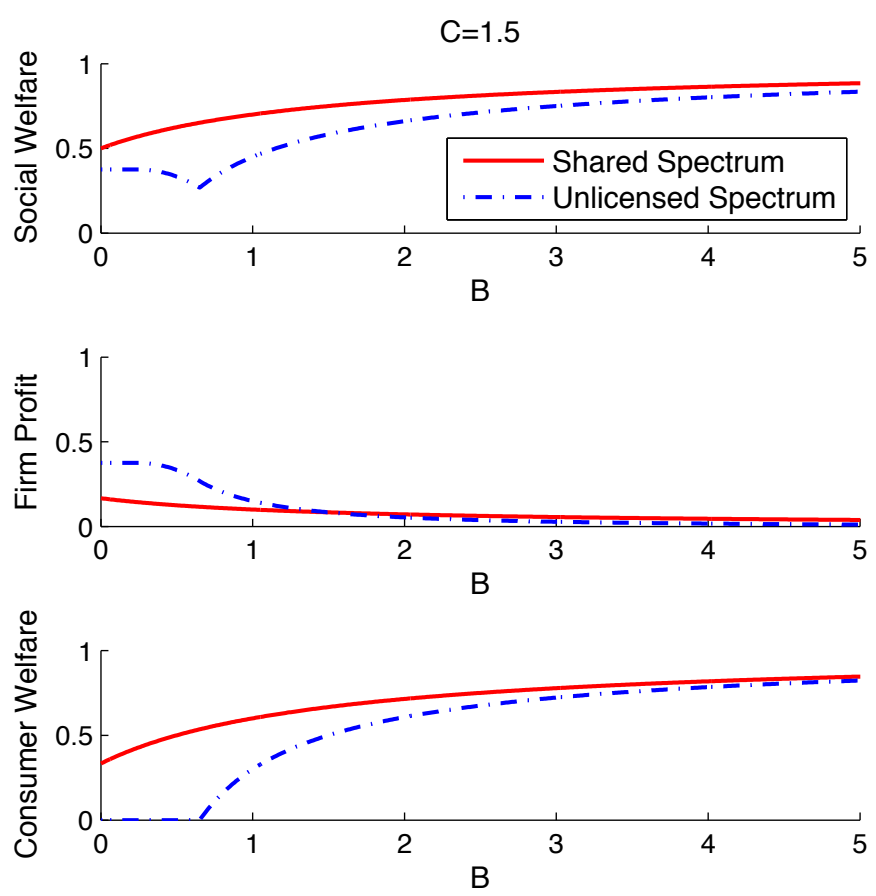

Fig. 6. Welfare comparison between sharing and unlicensed access with additional bandwidth $B$ when $C=1.5$.
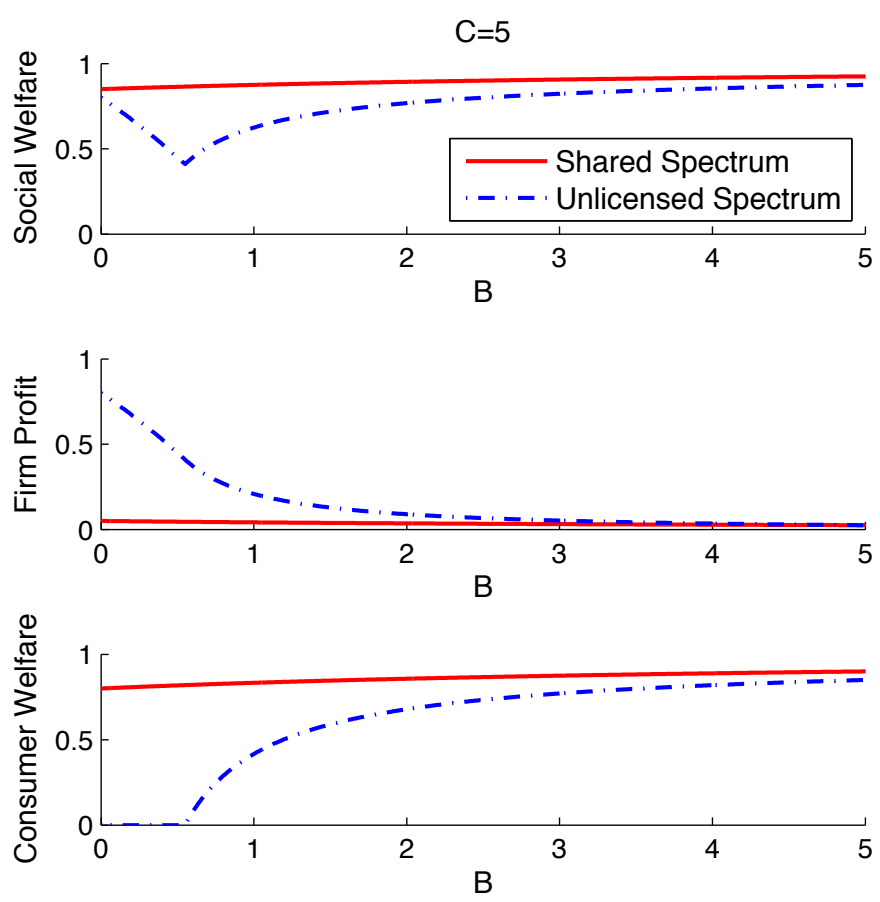

Fig. 7. Welfare comparison between sharing and unlicensed access with additional bandwidth $B$ when $C=5$.

main result, stated next, shows that the main insights from the previous section still apply in this general case.

Theorem 4.1: Social welfare and consumer welfare with shared spectrum grows with additional bandwidth $B$ and are no less than with unlicensed spectrum, which may decrease within a certain range and then increase with $B$. When $B$ is large enough, sharing provides the incumbent more profit than unlicensed access.

Proof: First we prove the consumer welfare with sharing is no less than with unlicensed access. As consumer welfare is the area above the delivered price and under $P(x)$, we want to show $g\left(\frac{x^{\prime}-x_{1}}{B}\right)>g\left(\frac{x^{*}}{(B+C}\right)$, i.e., $x^{\prime}<x^{*}$ as in Fig. 8. Suppose not, that is $g\left(\frac{x^{\prime}-x_{1}}{B}\right) \leq g\left(\frac{x^{*}}{(B+C}\right)$, i.e., $x^{\prime} \geq x^{*}$. Then we have

$$
\frac{x^{\prime}-x_{1}}{B} \leq \frac{x^{*}}{B+C} \leq \frac{x^{\prime}}{B+C} .
$$

The left inequality follows since $g$ is strictly increasing while the right inequality results from our assumption that $x^{\prime} \geq x^{*}$. From the first and third term in (3), we obtain $x_{1} \geq \frac{C x^{\prime}}{B+C}$. Since $g(x / C)$ is increasing, this implies that

$$
g\left(x_{1} / C\right) \geq g\left(x^{\prime} /(B+C)\right) .
$$

Likewise, $g\left(x^{\prime} /(B+C)\right) \geq g\left(x^{*} /(B+C)\right)$ and $g\left(x^{*} /(B+\right.$ $C)) \geq g\left(\left(x^{\prime}-x_{1}\right) / B\right)$, combining these with (4), we have $g\left(x_{1} / C\right) \geq g\left(\left(x^{\prime}-x_{1}\right) / B\right)$. This shows the congestion of the primary firm with unlicensed spectrum $g\left(x_{1} / C\right)$ is no less than the delivered price $g\left(\left(x^{\prime}-x_{1}\right) / B\right)$, in which case the primary SP will have zero or negative firm profit. This can not be an equilibrium as the primary SP can always find a price at which it makes positive profit.

Next, we will show that the social welfare with sharing is no less than that with unlicensed access. We have already shown that $P\left(x^{*}\right)<P\left(x^{\prime}\right)$. As shown in Fig. 8, this implies that consumer welfare with shared spectrum is larger than the sum of the consumer welfare with unlicensed spectrum and the partial firm profit with unlicensed spectrum corresponding to the area above the horizontal line $P\left(x^{*}\right)$, which is shown as the square area $D-C-E-F$ in Figure 8 . The firm profit with sharing is the largest square area in the region bounded by the curve $g(x /(B+C))$ and the horizontal line $P\left(x^{*}\right)$. Note the remaining part of firm profit with unlicensed spectrum lies in this region, i.e., the square area $A-B-C-D$. Since social welfare is the sum of consumer welfare and firm profit, it then follows that the total social welfare of sharing is greater than with unlicensed access.

Finally, note that as $B$ grows large $g\left(x^{*} /(B+C)\right.$ and $g\left(x^{\prime}-\right.$ $\left.x_{1}\right) / B$ approach zero. In other words, the delivered price in both cases is approaching zero and so the firm profit in each case must also be approaching zero. Since, $x^{*}$ is bound, for large $B, g(x /(B+C))$ can be approximated by its first-order Taylor series at the origin. Using this, it can be shown that with sharing, as $B$ increases, firm profit approaches zero at a rate of $g^{\prime}(0) / 4(B+C)$. Likewise, with unlicensed spectrum, it can be shown that firm profit approaches zero at a rate upper bounded by $C g^{\prime}(0) / B^{2}$. Hence, for large enough $B$, firm profit with unlicensed spectrum must be less than that with sharing.

To illustrate this result we consider an example with linear congestion $g(x)=x$ and linear inverse demand $P(x)=1-x$. In this case, the equilibrium behavior can be derived in closed 


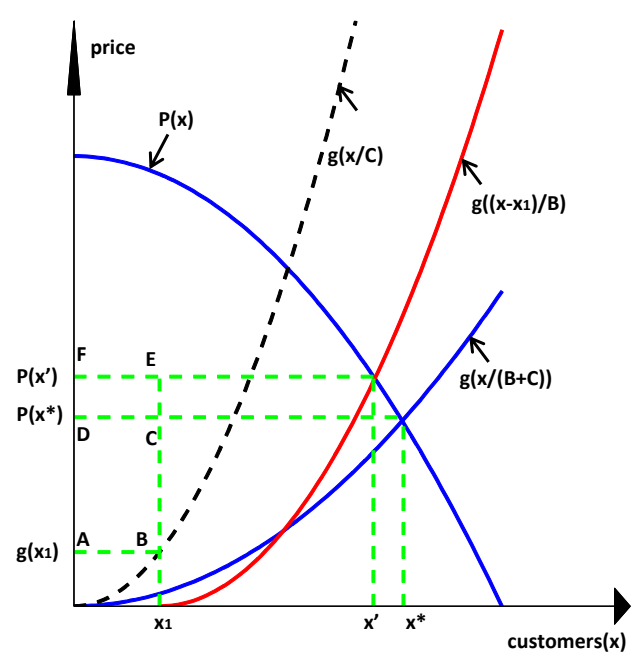

Fig. 8. Welfare comparison between sharing and unlicensed access: $x_{1}$ is the customer mass served by the primary firm with unlicensed access. $x^{*}$ is the total customer mass with sharing. $x^{\prime}$ is the total customer mass with unlicensed access.

form. Figure 9 shows the consumer welfare, total welfare and firm profit as a function of $B$, assuming that $C=1$. As both congestion cost and inverse demand are continuous, we see a smoother trend than in the case of homogeneous inversedemand. As stated in the theorem, both sharing and unlicensed access have increasing social welfare as $B$ grows while the welfare with sharing always stays above that with unlicensed access. With unlicensed access, the primary firm earns more profit than with sharing with small $B$ but its profit drops below that with sharing as $B$ increases. This is due to the large shift of traffic from the primary firm to unlicensed spectrum when more spectrum is available there. It also shows that with more spectrum available, firms tend to gain less profit. This in turn benefits more consumers as consumer welfare always increases with extra spectrum.

\section{Conclusions}

In this paper we have compared the impact of increasing competition in a wireless market by either adding shared or unlicensed spectrum. The main result is that sharing provides more social welfare and consumer welfare compared to unlicensed access. With large enough additional spectrum, sharing provides firms more profit as well. We provided a detailed discussion with linear congestion costs and homogeneous demands, showing that the gap between social welfare with shared and unlicensed spectrum is associated with the Braess's Paradox identified in earlier work. We then showed that this advantage of shared versus unlicensed access holds in a general model as well.

Our comparison of shared and unlicensed spectrum is based on a model where both primary and secondary users experience the same congestion function $g(\cdot)$ although they "see" different traffic. One direction of future work is to generalize to allow asymmetric congestion for primary and secondary
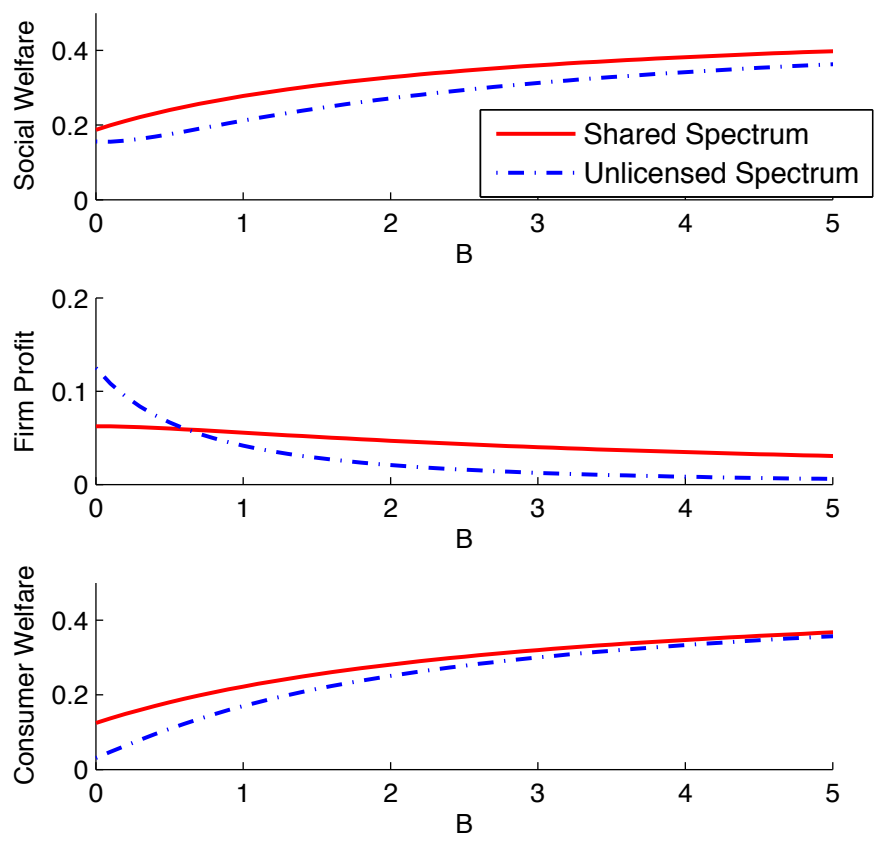

Fig. 9. Welfare comparison between sharing and unlicensed access when there is an additional spectrum of bandwidth $B$ with initial bandwidth $C=1$.

users. For example, with shared spectrum, secondary users could experience more congestion than primary users due to sensing delays. In that scenario, sharing may not always create more social welfare. Also more detailed models that better reflect specific cognitive communication schemes could be considered.

\section{REFERENCES}

[1] Cabric, D., S. Mishra, and R. Brodersen, "Implementation issues in spectrum sensing for cognitive radios," 2004 IEEE Asilomar Conference on Signals, systems and computers, 2004.

[2] D. Gurney, G. Buchwald, L. Ecklund, S.L. Kuffner, J. Grosspietsch, ”GeoLocation Database Techniques for Incumbent Protection in the TV White Space", IEEE DySPAN 2008.

[3] J. Huang, R. Berry, and M. Honig, "Auction-based spectrum sharing," ACM Mobile Networks and Applications, vol. 11, no. 3, pp. 405-418, June 2006.

[4] R. Saruthirathanaworakun and J.M. Peha, "Dynamic Primary-Secondary Spectrum Sharing with Cellular Systems," IEEE Crowncom 2010.

[5] C. Liu and R. Berry, "Competition with Shared Spectrum," 2014 IEEE DySPAN, Apr. 2014.

[6] T. Nguyen, H. Zhou. R. Berry, M. Honig, and R. Vohra, "The impact of additional unlicensed spectrum on wireless services competition," 2012 IEEE DySPAN, pp. 146-155, May 2011.

[7] D. Acemoglu, A. Ozdaglar, "Competition and efficiency in congested markets," Mathematics of Operations Research, Feb. 2007.

[8] R. Johari, G. Y. Weintraub, B. V. Roy, "Investment and market structure in industries with congestion". Operations Research, Sept. 2010.

[9] T. Nguyen, H. Zhou. R. Berry, M. Honig, and R. Vohra,"The price of free spectrum to heterogeneous users," in Workshop on the Economics of Networks, Systems and Computation(NetEcon'11), 2011.

[10] H. Zhou, R. Berry, M. Honig, R. Vohra, "Investment and competition in unlicensed spectrum", 46th Annual Conference on Information Sciences and Systems(CISS), 2012

[11] J. G. Wardrop, "Some theoretical aspects of road traffic research," Proceedings, Institute of Civil Engineers, PART II, vol. 1, pp. 325378, 1952.

[12] D. Braess, Unternehmensforschung, vol. 12, pp. 258-268, 1968 